\title{
The impact of the angioplasty of the renal artery and cold ischemia time in kidney transplantation on graft function
}

\author{
Zavacka $\mathrm{M}^{1}$, Frankovicova $\mathrm{M}^{1}$, Pobehova $\mathrm{J}^{1}$, Zavacky $\mathrm{P}^{2}$ \\ 1st Surgery Clinic, L. Pasteur University Hospital, Kosice, Slovakia. peterzavacky963@gmail.com
}

\begin{abstract}
The aim of this study was to analyze the benefit of the patients after renal transplantation with an assessment of the significance of different surgical techniques in patients with renal transplantation (not only from the dead but also the living donors) with multiple arteries. 457 patients with end stage renal disease (ESRD) in the treatment using the extracorporeal elimination method (haemodialysis, or peritoneal dialysis), who in the period from 2005 to 2015 had a kidney transplant were included in our retrospective study. Our results confirm that the patients after kidney transplantation with cold ischemia time more than 12 hours have 2.5 times higher risk of delayed onset, possibly failure of graft function compared to those with cold ischemia time less than 12 hours. This confirms our experience that the best option for the patient to achieve a stable graft function with long-term perspective is cold ischemia time of less than 12 hours and the realisation of renal artery angioplasty. In this case, the risk of delayed onset of transplanted kidney function or graft failure decreases 4.5 times compared to the respondents with cold ischemia time more than 12 hours without carrying out arterial angioplasty (Tab. 1, Fig. 4, Ref. 16). Text in PDF www.elis.sk.

KEY WORDS: kidney transplantation, vascular anastomosis, angioplasty, graft function failure, cold ischemic time.
\end{abstract}

\section{Introduction}

Transplantation compared to the other methods of renal function replacement provides to patients with renal failure the highest possible quality of life while also being economically the cheapest way of treatment of renal failure with ESRD (end stage renal disease) (Port, 1993; Wolfe, 1999; Seghal, 2002).

\section{Vascular graft in transplant surgery}

The patency of the vascular graft is dependent on the quality of the vascular connections. Poor quality of the vessel wall, the intima damage, media snagging, dissection, contusion and subintimal haematoma are the indications for the vessel resection. Properly everted intima, the average knot traction, sufficiently large engagement of the wall, it all depends on the clinical experience of the operational erudition.

Mohamed et al. describes the renal vascular reconstruction in kidney transplants using polytetrafluoroethylene (PTFE) graft (Mohamed H., 2007). Up to now, however, no studies comparing long-term outcomes of biological vs. prosthetic vascular grafts in kidney transplantation are known. The various techniques for the

${ }^{1}$ Clinic of Vascular Surgery, East Slovak Institute of Cardiovascular Diseases, Inc., Kosice, Slovakia, ${ }^{2}$ Ist Clinic of Surgery, University Hospital Louis Pasteur, Kosice, Slovakia

Address for correspondence: P. Zavacky, MD, PhD, MPH, 1st Surgery Clinic, L. Pasteur University Hospital, Trieda SNP 1, SK-040 11 Kosice, Slovakia. repair of short or damaged donor renal artery include an end-to-end anastomosis of artery graft, side-to-side anastomosis of branched artery, and use of iliacal arterial graft. Short donor renal vein can be adjusted by transferring the recipient's external iliac vein after ligation of internal iliac vein, extending the donor vein by dissection in the renal hilum and the use of venous grafts.

\section{The types of adjustments of vascular pedicle:}

1. Prolongation of the iliac artery graft

2. Revision of aortic patch with double kidney artery

3. Repairing of smaller damaged arteries: re-implantation into greater renal vessel or sewn by end to end to the donor vascular graft 4. Combination of types $1-3$

\section{Methods}

Four hundred and fifty seven patients with ESRD (end stage renal disease) in the treatment using the extracorporeal elimination method (haemodialysis, or peritoneal dialysis), who, between 2005 and 2015 underwent a kidney transplantation at the First Department of Surgery of UHLP and Medical School, University of P.J. Safarik in Kosice were included in our retrospective observational cohort study. Classifying criterion was successfully implemented kidney transplant. Exclusion criteria were death, graftectomy and acute heart failure within 24 hours after kidney transplantation (KTx).

The kidney was transplanted from a dead or living donor. In each one we controlled: anatomical structures, the integrity of the 


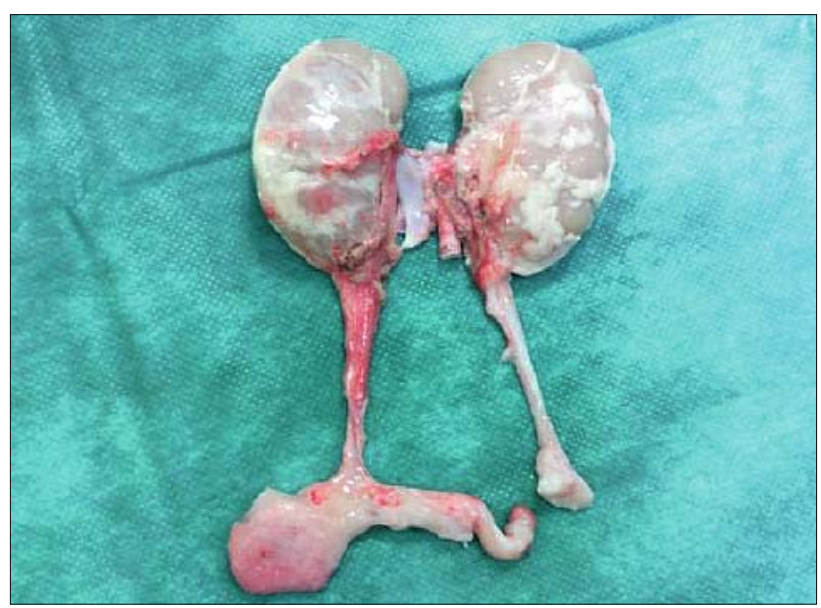

Fig. 1. Donors kidneys before transplantation (source: 1st Surgical Clinic, UNLP).

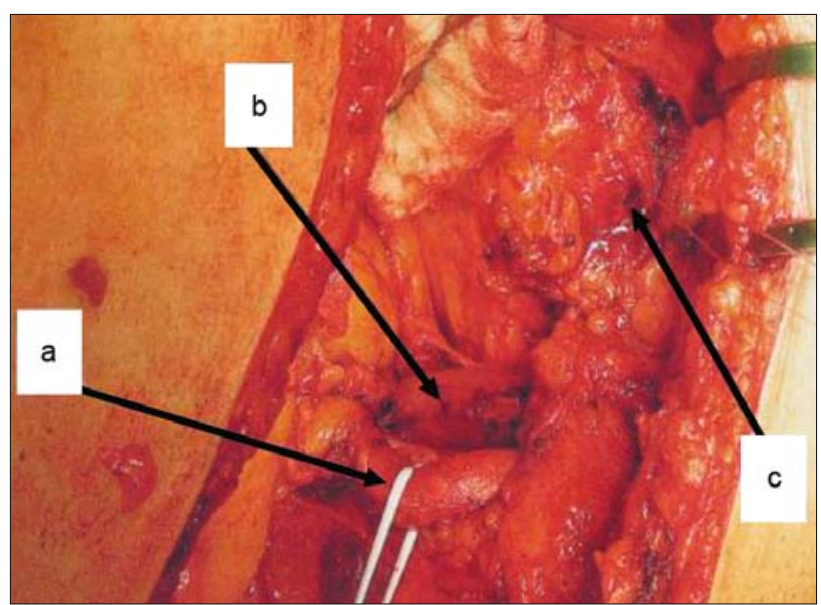

Fig. 2. Dissected vasa iliaca externa: a - a. ilica, $b-v$. iliaca, $c$ - bladder (Source: Ist Surgical Clinic, UNLP).

intima of arteries and veins, the presence of accessory arteries, rinsing efficiency (see Fig. 1). If the accessory arteries were not damaged and were not far from the main arteries, we left them on the same target. If they were distant, damaged, or separated, we sewed them into the main artery "end to side", or have them rebuilt by vascular allografts (usually iliac vessels), or autografts taken from the recipient (arteria epigastrica inferior, or vena saphena magna).

Kidney was inserted extraperitoneally into the iliac fossa, where the donor vessels were sewn end-to-side on the iliac arteries of the recipient following the non-absorbable suture material. We started with vein anastomosis, and after its checking followed the connection of arteries to a. iliaca interna, or a. iliaca externa (see Fig. 2). At sclerotic changes on the artery the anastomosis was sewn on at the place with the least disability. If this was not possible, we performed endarterectomy of the given part and then we sewed the anastomosis.

In the next step we reconstructed the urinary tract. After isolation of a part of the anterior and lateral wall of the bladder, laterally from vertex we implanted the ureter of the transplanted kidney into the bladder.

\section{Results}

The research cohort consisted of 457 patients who underwent a kidney transplantation (from a living or deceased donor) with a mean age of $47.92( \pm 12.22)$ years (range $9-75)$. In the set the males were predominant with the percentage of $58.4 \%$ (267), females were $41.6 \%$ (190). Figure 1 shows the characteristics of set.

Arterial angioplasty was performed in $22.3 \%$ (102) of the patients, out of which the arteries individually sewn on were in $5.5 \%$ (25), on the common target $9.4 \%$ (43), and in $7.4 \%$ (34) angioplasty was carried out as an implantation of pole renal arteries into the main strain.

\section{The values of urea and creatinine in the relation to the onset type of graft function}

Creatinine and urea values measured before surgery were not statistically significantly different (either as a continuous or as a categorical variable), according to the onset type of graft function. The opposite situation occurred in these values at the emission of the patients. The later onset of graft function, or its failure is also reflected in the significantly different (worse) values of urea and creatinine at patient release from the hospital $(\mathrm{p}<0.001)$ (Tab. 1).

Tab. 1. A comparison of mean value of creatinine and urea before transplantation and after discharge in the group of patients with immediate, delayed onset, or without the onset of graft function (ANOVA).

\begin{tabular}{|c|c|c|c|c|}
\hline & The onset of graft function & $\mathrm{n}$ & Mean (SD) & ANOVA \\
\hline \multirow{3}{*}{ Creatinine value before $\mathrm{Tx}$} & immediately & 266 & $597.7(246.9)$ & $F=0.676$ \\
\hline & delayed & 171 & $622.0(221.2)$ & $\mathrm{p}=0.509$ \\
\hline & without onset & 20 & $634.3(211.3)$ & $\mathrm{NS}$ \\
\hline \multirow{3}{*}{ Creatinine value after $\mathrm{Tx}$} & immediately & 266 & $136.4(35.1)$ & $F=306.857$ \\
\hline & delayed & 171 & $204.9(115.1)$ & $\mathrm{p}=0.000$ \\
\hline & without onset & 18 & $665.8(242.2)$ & $\mathrm{p}<0.001$ \\
\hline \multirow{3}{*}{ Value of urea before Tx } & immediately & 266 & $14.7(6.5)$ & $\mathrm{F}=0.781$ \\
\hline & delayed & 171 & $14.9(6.2)$ & $\mathrm{p}=0.58$ \\
\hline & without onset & 20 & $16.6(8.0)$ & NS \\
\hline \multirow{3}{*}{ Value of urea adter Tx } & immediately & 266 & $8.8(3.6)$ & $F=46.918$ \\
\hline & delayed & 171 & $11.8(4.6)$ & $\mathrm{p}=0.000$ \\
\hline & without onset & 18 & $16.2(7.3)$ & $\mathrm{p}<0.001$ \\
\hline
\end{tabular}




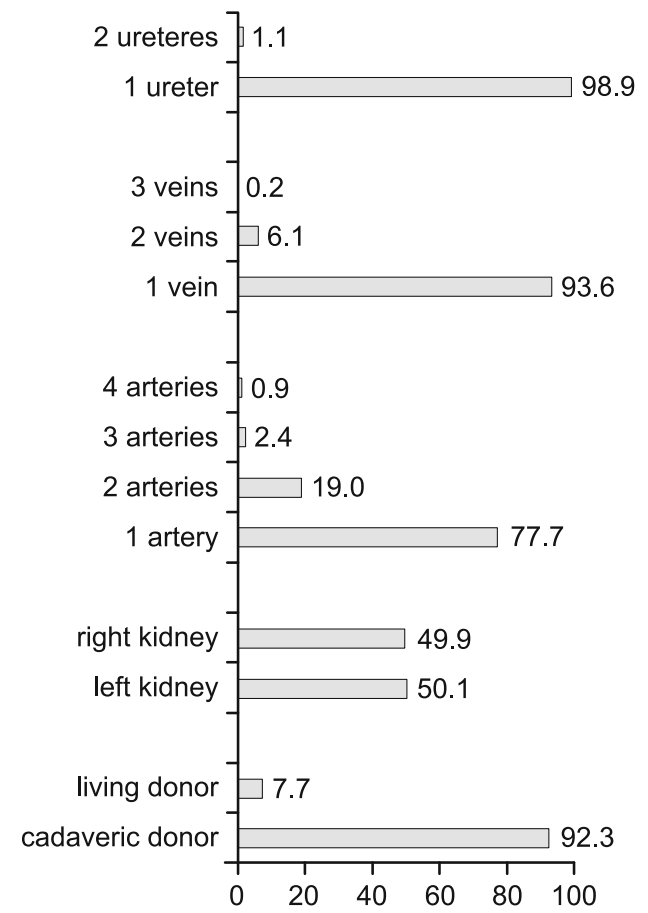

Fig. 3. The characteristics of set.

Similarly, the patient groups did not differ significantly for the type of surgery and time of the cold ischemia in the values of creatinine after transplantation. However, for comparison of the patients according to the type of surgery and the time of cold ischemia, we confirmed a statistically significant difference in the values of urea after surgery. The group without angioplasty with cold ischemia time more than 12 hours had significantly higher values of urea at their release from hospital compared to the group of patients without angioplasty, but transplantation was carried out to 12 hours $(\mathrm{p}<0.05)$.

Causality of the type of surgical intervention, gender, or age with the risk of delayed onset or the onset, or failure of graft function have not been confirmed. On the other hand, the longer period of cold ischemia was significantly associated with the onset of graft function. Transplanted patients with cold ischemia time more than 12 hours had a 2 and a half times higher chance $(\mathrm{OR}=2.49$ / CI: 1.50 to 4.13 ) of delayed onset, or failure of graft function compared to those with cold ischemia time shorter than 12 hours (Fig. 2).

\section{The type of the surgery and the onset of graft function}

The patients without angioplasty, but cold ischemia time was up to 12 hours had more than twice lower chance (OR / CI: 0.43 / 0.25 to 0.75 ) of delayed onset, or failure of graft function compared to the respondents with cold ischemia time more than 12 hours, but without necessity of performing angioplasty. Similarly, patients with cold ischemia time of less than 12 hours who underwent angioplasty had four and a half times lower chance (OR / CI: $0.22 / 0.06-0.79$ ) of delayed onset, or failure of graft function compared to those with cold ischemia time more than 12 hours, but without necessity of carrying out angioplasty.

The type of the surgery, duration of the cold ischemia, comorbidity and the onset of graft function

The patients with cold ischemia time more than 12 hours had 2.42 times higher probability, and the patients with co-morbidity (CAD / DM) had more likely 1.65 times delayed start function, or graft failure compared to those with shorter cold ischemia and without the co-morbidity. Interaction of the influence of the type of surgery and comorbidities was not statistically significant. In other words, the type of surgery was not associated significantly with the onset of graft function, even when it was evaluated separately in the group with and without comorbidity.

\section{Discussion}

The aim of this study was to analyze the benefit of the renal transplant patients with an evaluation of the significance of the different surgical techniques. We followed up 457 patients who underwent kidney transplantation during 2005-2015 at the First Surgical Clinic UHLP and the Medical Faculty of the University of P.J. Safarik in Kosice retrospectively.

Anatomically, there were most of the kidneys with 1 artery 1 (77.7\%), $19 \%$ with two arteries, $2.4 \%$ with 3 arteries, and 0.7 $\%$ with 4 arteries. The overwhelming number - in $93.6 \% 1$ vein was present, $6.1 \%$ of donor kidney had 2 veins, and only $0.2 \%$ of donated kidneys had 3 veins. Only in $1.1 \%$ of the patients there were with 2 ureters on the donor kidney. A lot of authors report

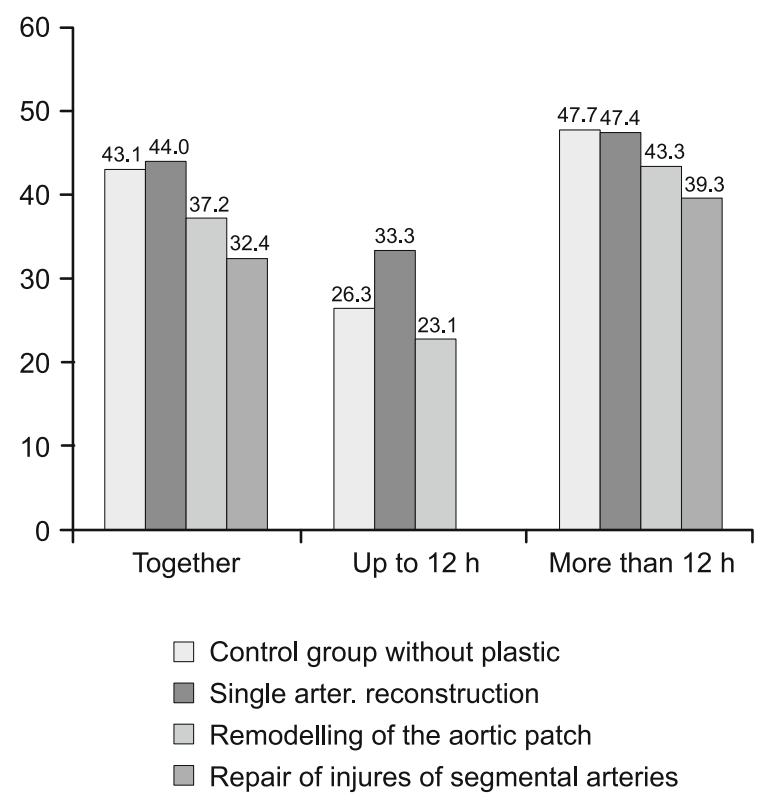

Fig. 4. The occurrence of delayed onset, or failure of the onset of graft function in the patient groups according to the type of surgical procedure, and the time of cold ischemia (in \%). 
the incidence of multiple renal arteries in the range of $18 \%$ to 30 \% (Suthanthiran et al, 1994; Navrátil et al, 2005; Viklický et al, 2008). The incidence in the patients involved in the Aydin's study from 2014 with renal transplantations was $12.8 \%$. The authors retrospectively evaluated 225 adult kidney transplants. 29 patients $(12.8 \%)$ had grafts with multiple renal arteries. For reconstruction of multiple renal arteries Aydin et al. describe various techniques (Aydin et al, 2004). So far however, none of the reconstruction techniques has been shown to be the best, referring to complications and graft survival rate. Neither this study showed any significant differences in grafts and patients between the allograft with multiple and a single artery. When it was appropriate, Aydin et al preferred connection of the artery extracorporeally as one renal artery in order to create a common disk. In the allografts with multiple renal arteries no significant differences were demonstrated in the graft survival in the patients with different reconstruction techniques. The mean systolic blood pressure, creatinine values in the first year and the last observation, and complication rate were in the acceptable range (Aydin et al, 2004).

The values of urea and creatinine reflect the renal function (Jafri et al, 2009). Therefore, when we consider the fact that patients undergoing kidney transplantation are in the final stages of CKD in need of renal replacement therapy, the analysis results did not confirm adequately the relationship between these values and the onset of graft function (Seghal, 2002). However, after the successful transplantation, at the time of the patient release, the parameters of kidney functioning are significantly associated with the onset of graft function, since there is a linear relationship between them (Jafri et al, 2009). This is related to the confirmation of a statistically significant inverse association between later onset of graft function, or its failure and higher levels of urea and creatinine at the time of release. Statistically significant association have also been demonstrated between the values of urea and creatinine and the type of surgery. After comparison of the groups of patients according to the type of plastic we confirmed a statistically significant difference in the values of urea after kidney transplantation. Namely the group of patients transplanted with the cold ischemia time more than 12 hours had significantly higher value of urea at their release, compared with patients without angioplasty, but the cold ischemia time of the kidney was less than 12 hours $(\mathrm{p}<0.05)$.

In this work we have also confirmed the significance of performing the reconstruction of blood vessels in the kidneys of donors, which was not associated with gender and age, but with the level of urea. The group without angioplasty, with cold ischemia time more than 12 hours had significantly higher values of urea at their release compared to the group of patients without angioplasty, but the transplantation was performed within 12 hours. On the other hand, the association between the level of creatinine at release and the type of surgical procedure was confirmed only in the group of patients with severe comorbidity in terms of ischaemic heart disease and/or diabetes.

Moreover, we have shown the link between a longer period of cold ischaemia and the onset of graft function. The patients after kidney transplantation with cold ischemia time more than 12 hours had 2.5 times higher risk of delayed onset, or failure of graft function compared to those with cold ischemia time less than 12 hours. The patients without plastic, but the cold ischemic time was up to 12 hours, had more than twice lower risk of delayed onset, or failure of graft function compared to respondents with cold ischemia time longer than 12 hours, but without a need of plastic. This result explains the reasons for shortening of the cold ischemia time to the minimum.

However, isolated vessel plastic was not independently associated with the graft function at the evaluation without cold ischemia time and comorbidities. These results offer an ideal option that is: the cold ischemia time under 12 hours occurring concurrently, and performing the renal artery angioplasty. In this case, the risk of delayed onset of the transplanted kidney function, or graft failure decreases 4.5 times compared to the respondents with the cold ischemia time above 12 hours without angioplasty. Based upon the analyses the important information follows which is profiting from the reconstruction of donor kidney arteries, while the benefit rises more in the patients with co-morbidities than without them (Levey 2010, Sis, 2010). On the other hand, not only the very long period of cold ischemia and non-implementation of angioplasty of the donor kidney if there are options for it, increase the risk of delayed onset, or loss of graft function as well as co-morbidity. Patients with co-morbidity in terms of ischemic heart disease and diabetes of first and/or second type had almost a 2 times higher probability of delayed onset of graft function, or its failure compared to those with shorter cold ischemia time and without comorbidity. An association among the renal function (measured according to the serum creatinine values) after the first year from kidney transplantation, and carrying out the plastic, and/or shorter ischemia time has not been confirmed. To these results testifies the fact that after stabilisation period of three months the aspect of surgical intervention does not come into focus. For the given period, adequate immunosuppression, the absence of infection, rejection, and other complications are the important factors for preservation of the kidney function (Abecassis, 2008). The results in connection with a statistically significant association between male sex and impaired graft were confirmed by several authors (Franke 2003; Benjamins, 2004). In our set, we demonstrated that men in comparison with women have approximately 2 times higher chance of deterioration of the graft function after 3 months and after 1 year from kidney transplantation.

Statistically significant differences were confirmed only in the control groups without angioplasty with different cold ischemia time in month 3 after transplantation. The control group without angioplasty with the cold ischemia time up to 12 hours had significantly lower mean values of creatinine after 3 months from the transplantation in comparison with the control group with the cold ischemia time more than 12 hours.

Despite the fact that no other significant association was demonstrated between the graft function and performing angioplasty, or cold ischemia time after the first year from the kidney transplantation, based upon the results the trend of the development of creatinine level was recorded, while the lowest mean values after 3 months had the patients with the cold ischemia time up to 12 hours and had only one artery, or without angioplasty. 
416-420

\section{Conclusion}

In the study we confirmed the significance of performing the reconstruction of blood vessels in the donor kidneys that was not associated with sex and age, but with the serum urea level. The strong point of the work is comprehensive compilation of transplanted patients in the period of ten years within one transplant centre in Slovakia, with the majority carrying out kidney transplantation. Longitudinality of the data processing strengthens the meaningfulness of the results.

\section{References}

1. Abecassis M, Barlett ST, Collins AJ et al. Kidney transplantation as primary therapy for end-stage renal disease: A National Kidney Foundation/ Kidney Disease Outcomes Quality Initiative (NKF/KDOQITM) Conference. Clin J Am Soc Nephrol 2008; 3: 471-480.

2. Aydin NM, Berber I, Altaca G et al. The outcome of kidney transplants with multiple renal arteries. BMC Surg 2004; 4: 4-8.

3. Benjamins MR, Hummer RA, Eberstein IW, Nam CB. Self-reported health and adult mortality risk: An analysis of cause-specific mortality. Soc Sci Med 2004; 59: 1297-1306.

4. Franke GH, Reimer J, Philipp T, Heemann U. Aspects of quality of life through end-stage renal disease. Qual Life Res 2003; 12: 103-115.

5. Jafri SSA, Younas M, Chughtai MN et al. Surgical aspect and outcomes of Kidney transplantation with multiple renal arteries. Annals 2009; 15 (2): $122-128$.

6. Levey AS, Stevens LA. Estimating GFR using the CKD Epidemiology Collaboration (CKD-EPI) Creatinine Equation: More Accurate GFR Estimates, Lower CKD Prevalence Estimates, and Better Risk Predictions. Am J Kidney Dis 2010; 55: 622-627.
7. Mohamed HK, Anil AT, Ponusamy MH et al. Renal vessel reconstruction in kidney transplantation using a polytetrafluor ethylene (PTFE) vascular graft. J Surg Case Rep 2007; 22: 1030-1032.

8. Navrátil P. Praktická urológia u nemocných v dialyzační léčbe, před a po transplantaci ledviny. Hradec Králové: Ol'ga Čermáková, 2005, 67-69.

9. Petrášová D, Chmelárová A, Kuchta M. Immunomodulation effect of imunoglucan on oxidant-antioxidative system in experiment. Physiol Res 2007; 56: 28.

10. Port FK, Wolfe RA, Mauger EA et al. Comparison of survival probabilities for dialysis patients vs cadaveric renal transplant recipients. J Amer Med Ass 1993; 270: 1339-1343.

11. Seghal AR. What is the best treatment for end-stage renal disease? Am J Med 2002; 112: 735-736.

12. Sis B, Mengel M., Haas M et al. Banff ' 09 meeting report: Antibody mediated graft deterioration and implementation of banff working groups. Am J Transplant 2010; 10: 464-471.

13. Suthanthiran M, Strom TB. Renal transplantation. N Engl J Med 1994; 331: 365-376.

14. Viklický O, Janoušek L, Baláž P et al. Transplantace ledviny v klinické praxi. Praha: Grada, 2008, 45-55.

15. Vol'anská $M$ et al. Angiogenesis and endothelin of critical limb ischaemia. Physiol Res 2007; 56: 39.

16. Wolfe RA, Ashby VB, Milford EL et al. Comparison of mortality in all patients on dialysis, patients on dialysis awaiting transplantation, and recipients of a first cadaveric transplant. N Engl J Med 1999; 341 (23): 1725-1730.

Received February 23, 2018. Accepted March 27, 2018. 